Work in the Department of Organic Chemistry has been reoriented considerably. In the study of diterpenoids a new general method has been developed by the synthesis of tricyclic $\mathrm{C}_{1}$-ketones. A new stereo-specific synthesis of $d l$-homocamphoric acid has been developed from camphononic acid and a similar synthesis of $d l$-homoapocamphoric acid completed. Efforts are also being made to develop a stereospecific synthesis of a steroidal nucleus, and the technique using liquid ammonia for the preparation of intermediates has been improved. A synthesis of the dicarboxylic acid, $\mathrm{C}_{12} \mathrm{H}_{14} \mathrm{O}_{4}$, obtained by the degradation of picrotoxin, has been achieved, and the ultra-violet absorption spectra of benzo- $\alpha$ - and $\gamma$-pyronos correlated with the corresponding chromophoric structures.

The Department of Inorganic Chemistry has been concerned chiefly with the chemistry of co-ordination complexes, especially the cis-trans isomerism of copper complexes, and with the use of organic reagents for inorganic analysis. A critical review is in progress of colorimetric methods for the determination of titanium, chromium, uranium and palladium, and a new method has been developed by the colorimetric determination of titanium with salicylhydroxamic acid, while salicylamidoxime has been used for the gravimetric determination of transitional elements, as well as for their separation and the colorimetric determination of uranium and iron.

\section{LAW OF RED SHIFTS AND THE DISTANCES OF NEBULA}

THE George Darwin Lecture of the Royal Astronomical Society was delivered by the late Dr. Edwin Hubble on May 8, 1953, in the Society's rooms at Burlington House, London, the subject of the Lecture being the law of red shifts-the correlation between distances of nebulæ and displacements in their spectra*. Three phases in the history of the law of red shifts were discussed; the first, the discovery phase, commenced with Slipher's measurement in 1912 of the velocity of $M 31$, which he found to $\mathrm{be}-300 \mathrm{~km}$. $/ \mathrm{sec}$., and during the next ten years he contributed forty-two out of forty-six nebular velocities then available. The list was completely dominated by large positive velocities which ranged up to $1,800 \mathrm{~km}$. $/ \mathrm{sec}$. in the case of $N G C 584$.

Attempts from 1916 onwards to derive the solar motion with respect to the nebulæ were frustrated by the positive signs indicating general recession, but improvements in the results followed Wirtz's introduction of a constant $K$ term in the equations. Although the improvement was not sufficient to render the results acceptable, it was seen that the next logical step would be to replace the constant $K$ by $r K$, that is, by a term which varied with the distances of the individual nebulæ; and during the early and middle twenties various solutions were made, measures of relative distances being based on apparent luminosities and apparent diameters. Failure was due, as appeared later, to the relatively short range covered by the radial velocities being insufficient to give reliable individual distances. The problem was finally solved when the brightest stars in late-type spirals and irregular nebulæ were established as suitable indicators of distance and

* Published in Mon. Not, Roy. Astro. Soc., 113, 6 (1953), and also, slightly condensed, in The Observatory, 73 , No. 874 (1953). were calibrated by Cepheids in a few of the nearest nebulæ. Using the upper limits of brightness of these stars as a distance criterion and applying it to the nebulæ with measured velocities, the law of velocitydistance shifts, now known as the law of red shifts, emerged approximately in its present form. This law was established in 1928 out to about seven million light-years, as an approximate linear relation according to which velocities of recession increased at the rate of the order of $500 \mathrm{~km}$. $/ \mathrm{sec}$. per million parsecs of distance, or about $150 \mathrm{~km}$./sec. per million light-years.

The second phase, a Mt. Wilson project, lasted from 1928 to 1936 , during which Humason assembled spectra of nebulæ and Hubble attempted to estimate their distances. For various reasons, attention was concentrated on clusters, and, when these could not be observed to advantage, spectra of field nebulæ, isolated or members of small groups, were assembled in large numbers; these data provided an account of red shifts in the nearer, more conspicuous nebulæ of all kinds. The results of the second phase were as follows. The law of red shifts appeared to be linear, and the red shifts increased at the rate of about $160 \mathrm{~km}$. $/ \mathrm{sec}$. per million light-years. The solar motion was largely accounted for by galactic rotation, and the residual represented the peculiar motion of the stellar system and was small and uncertain, of the order of 100-150 $\mathrm{km}$./sec. towards galactic latitude $+30^{\circ}$ approximately, and longitude in the quadrant $50^{\circ}-140^{\circ}$. 'The law of red shifts did not operate within the Local Group; and, lastly, magnitude-velocity relations, fitted to data for clusters, resolved nebulæ and field nebulæ, and displaced from one another by magnitude intervals indicating relative absolute luminosities, could be used to calibrate all the relations in terms of brightest stars.

In the interim between the second and third phases, Mayall began assembling spectra with a very fine ultra-violet spectrograph on the 36-in. Crossley at Mt. Hamilton, and gave special attention to the later-type spirals and other systems of low surface brightness for which his reflector was as efficient as the larger telescopes on Mt. Wilson. His list now numbers about 280 nebular velocities and with Humason's list, when the two are published, will total about 750 individual nebulæ. The data furnish most important information of every kind, and full discussion must await publication of the lists, though certain matters are divulged by Hubble; limits of space prevent anything more than reference to them in this article.

The completion of the 200 -in. reflector and its accessories initiated the third and current phase of the development, the programme of which has two parts, the first being the extension of the observed range. During the first season that a nebular spectrograph could be used on the 200-in. (1950-5I), Humason obtained spectra in three clusters which had been unsuccessfully tried with the 100-in.; one of these, the Hydra cluster, had a velocity of 61,000 $\mathrm{km}$. / sec. The 48-in. Schmidt reflector can be used to this limit, but beyond it clusters cannot be identified with certainty on the survey plates. Humason is confident, however, that velocitios up to $\mathbf{7 5 , 0 0 0}$ $\mathrm{km}$. /sec. at least can bo recorded when clusters have been located, but such locations would depend on purely chance finds with the 100 -in. and 200-in.

The second part of the programme deals with the revision of the distance scale. A review of the unit of distance has been pending for more than twenty 
years-after it was realized that many novæ and globular clusters in $M 31$ were fainter with respect to the Cepheids than was the case in our own system. It is now known that the classical Cepheids in $M 31$ are nearly 1.5 magnitudes brighter than was previously supposed ; in consequence, current estimates of absolute distances must be nearly doubled, and the so-called 'age of the universe' must be increased on the same scale. Among other steps in the programme are included the setting up of faint-magnitude sequences in Selected Areas, the measurement of nebular magnitudes, corrections for effects of red shift on apparent magnitudes, and the examination of the possibility of errors arising from inter-nebular obscuration and systematic evolution changes in luminosities during the travel time of the light from the nebulæ. Each of these is considered in logical order in the later part of the Lecture, which ends with a warning that "we are already in the region of diminishing returns"-in other words, instruments will be increasingly expensive and progress increasingly slow. So far as the immediate future is concerned, the most promising programmes accept the observable regions as now defined, and, while hoping for only modest extensions in space, concentrate on inereased precision and reliability in the recorded description. "The reconnaissance is being followed by an accurate survey; the explorations are pushed towards the next decimal place instead of the next cipher." The Lecture ends with an optimistic outlook: "From our home on the Earth, we look out into the distance and strive to imagine the sort of world into which we are born. Today we have reached far out into space. Our immediate neighbourhood we know rather intimately. But with increasing distance our knowledge fades, and fades rapidly, until at the last dim horizon we search among ghostly errors of observations for landmarks that are scarcely more substantial. The search will continue. The urge is older than history. It is not satisfied. and it will not be suppressed".

\section{ABSORPTION OF IONS BY PLANTS}

\author{
By D. REICHENBERG \\ Chemical Research Laboratory, Teddington \\ and J. F. SUTCLIFFE \\ Botany Department, King's College, London
}

$I^{\mathrm{T}}$ has been shown ${ }^{1}$ that in water culture some plants may absorb both cations and anions at a rate which is independent over a wide range of the totel concentration in the culture medium, provided their relative concentrations in the medium are kept fixed. Such results may be explained on the basis that the rate of absorption is limited either by the number of suitable 'carrier' molecules or by the amount of energy available from respiration ${ }^{2}$. How. ever, recent work has shown that ion exchange processes may follow a closely similar pattern. The rate of exchange of sodium for hydrogen on both strong- ${ }^{3}$ and weak-acid ${ }^{4}$ cation exchange resins has been shown to be independent of the sodium ion concentration in solution over a wide range. In this range, the rate-controlling process has been shown to be the diffusion of ions through the resin material, the constancy of rate being due to the fact that the boundary concentrations are independent of those in solution. It appears, therefore, that a simple alternative hypothesis for the uptake of ions by plants may be postulated on the basis of ion exchange.

The assumption must be made that there is present in plant cells a membrane (or. membranes) containing both anion and cation-exchange materials across which the movement of ions occurs. However, it is not necessary to postulate two entirely homofunctional substances, one completely acidic and the other completely basic; only that one is predominantly acidic and the other predominantly basic. The two might even be part of the same protein molecule provided the acidic and basic units were sufficiently segregated. The properties of such 'mosaic membranes' have been studied, both theoretically and experimentally, by Sollner ${ }^{5,8}$. To cause ion exchange to proceed it is necessary for the plant to provide on the inside of the membrane a continual supply of an appropriate electrolyte such as carbonic acid or some other weak organic acid. The cations would then be exchanged for hydrogen ions on the cation exchange material, and the anions likewise for bicarbonate (or other anion) on the anion exchanger. Thus the hydrogen ions and anions would diffuse separately through the cation and anion exchange materials respectively and recombine in the culture medium, while other ions from the medium would move in the opposite direction. It is known that hydrogen ions, bicarbonate and other anions are, in fact, yielded by plants to the external solution.

The energetics of transport by ion exchange diffusion are particularly simple and account readily for the fact that ions may be absorbed against a concentration gradient. Consider a cation exchange membrane separating infinite volumes of solutions 1 and 2 , in which the activities of sodium and hydrogen ions are, respectively, $\left[a_{\mathrm{Na}}\right]_{1},\left[a_{\mathrm{H}}\right]_{1},\left[a_{\mathrm{Na}}\right]_{2},\left[a_{\mathrm{H}}\right]_{2}$. 1 represents the culture medium and 2 the solution on the inside of the plant membrane. Sodium will diffuse through the membrane from solution 1 to solution 2 provided that the concentration of sodium ions in the membrane is greater on the side nearer solution 1. Since the uptake of sodium ions by cation exchange materials of both the strong-and weak-acid type has been shown to depend on the ratio $a_{\mathrm{Na}} / a_{\mathrm{H}}$ (if hydrogen is the competing ion), transport of sodium ions from solution 1 to solution 2 will occur if :

that is, if

$$
\frac{\left[a_{\mathrm{Na}}\right]_{1}}{\left[a_{\mathrm{H}}\right]_{1}}>\frac{\left[a_{\mathrm{Na}}\right]_{2}}{\left[a_{\mathrm{H}}\right]_{2}}
$$

$$
\frac{\left[a_{\mathrm{H}}\right]_{2}}{\left[a_{\mathrm{H}}\right]_{1}}>\frac{\left[a_{\mathrm{Na}}\right]_{2}}{\left[a_{\mathrm{Na}}\right]_{1}}
$$

Now consider the energetics of the process. Let $\left[a_{\mathrm{Na}}\right]_{1}$ be less than $\left[a_{\mathrm{Na}}\right]_{2}$. This means that sodium ions must be transported against a concentration gradient. For this an expenditure of free energy $R T \ln \frac{\left[a_{\mathrm{Na}}\right]_{2}}{\left[a_{\mathrm{Na}}\right]_{1}}$ per gm. ion is required. However, hydrogen ions will diffuse in the opposite direction assisted by their own concentration gradient which provides an amount of free energy $R T \ln \frac{\left[a_{\mathrm{H}}\right]_{2}}{\left[a_{\mathrm{H}}\right]_{1}}$ per 